\title{
Efectos del apoyo familiar, amigos y de escuela sobre el bullying y bienestar subjetivo en estudiantes de nivel secundario de Chile y Brasil
}

\author{
Effects of family, friends and school support about bullying and subjective \\ well-being from Chile and Brazil students
}

Rafael Miranda Ayala ${ }^{1,{ }^{*}, \text { Juan Carlos Oyanedel }}{ }^{2}$, Javier Torres ${ }^{3}$, ${ }^{1}$ Universidad Continental, ${ }^{2}$ Universidad Andrés Bello de Chile, ${ }^{3}$ Vallejos, INACAP Chile

\section{RESUMEN}

Este trabajo evalúa el efecto de los apoyos percibido por los estudiantes sobre las experiencias de bullying y el bienestar subjetivo; asimismo evalúa si la experiencia de bullying media la relación entre los apoyos percibidos y el bienestar subjetivo, para ello, se evaluaron a 1832 adolescentes de Chile y Brasil que forman parte de la muestra del Children's World. Análisis de correlaciones y modelo de ecuaciones estructurales fueron realizados, teniendo como resultados que los apoyos tienen un efecto significativo tanto para reducir el bullying como para promover el bienestar subjetivo en los estudiantes, sin embargo, no hay un efecto mediador del bullying sobre el bienestar subjetivo; asimismo para el caso de Chile $(b=-0,31, p<0,05)$ y Brasil $(b=-0,20$, $p<0,05$ ) el apoyo de la escuela es un predictor importante para reducir el bullying. Para el caso de bienestar subjetivo, el apoyo de los estudiantes $(b=$ $0,30, p<0,05)$ tiene una mayor influencia en Chile, en Brasil el apoyo de la escuela $(b=0,46, p<0,05)$ predice mejor el bienestar subjetivo; el apoyo social tiene un efecto positivo en el bienestar subjetivo de los niños y las niñas, tanto a nivel familiar, como escolar y de pares. Las diferencias del efecto que tienen estos tipos de apoyo que los participantes perciben como más importante son, el apoyo de la escuela, la familia y la de pares. En conclusión, los apoyos percibidos importan conforme a lo señalado en el modelo socio ecológico, tanto en el mediano plazo para reducir el bullying y a largo plazo para promover el bienestar subjetivo en los estudiantes.

Palabras claves: Bienestar subjetivo, bullying, apoyo social, apoyo de familia, apoyo de escuela, apoyo de pares.

\begin{abstract}
This work evaluates the effect of the social support perceived by students regarding bullying experiences and subjective well-being. Likewise, evaluates if the experience of bullying mediate the relationship between different perceived social supports and subjective well-being. Therefore evaluated 1832 adolescents in Chile and Brazil that are part of the sample of the Children's World. Structural equation model and correlation analysis were performed to evaluate the proposed objective. The results show that social supports matters. Most of the social supports reported have a significant effect to reduce bullying and promote subjective well-being at students. However, there is no effect mediator of bullying on subjective well-being. The results show that for the case of Chile $(b=-0,31, p<0,05)$ and Brazil (b $=-0,20, p<0,05)$ school support is a predictor for reducing bullying. For subjective well-being, school support $(b=0,30, p<0,05)$ has greater influence in Chile, in Brazil the school support $(b=0,46, p<$ $0,05)$ predicts better the subjective well-being. The results show that supports matter subject to state in the model ecological partner, in the medium term to reduce bullying and in the long run promote subjective well-being.
\end{abstract}

Keywords: Subjective well-being, bullying, social support, family support, school support, friend support

Historial del artículo:

Recibido, 14 de marzo 2017; aceptado, 22 de noviembre de 2017; disponible en línea, 05 de enero de 2018

* Correo: rmirandaa@continental.edu.pe

Este es un artículo de acceso abierto, licencia CC BY (https://creativecommons.org/licenses/by/4.0/) 


\section{INTRODUCCIÓN}

La violencia escolar es una pandemia mundial que afecta a uno de cuatro estudiantes (OMS, 2003) y representa un fenómeno que culturalmente se encuentra presente dentro del ambiente educativo. Olweus, en la década de 1970, fue el primero en estudiar de forma teórica dicho comportamiento. Hoy, este fenómeno es universalmente conocido como bullying, concepto que refiere a distintas situaciones de intimidación, acoso, abuso, hostigamiento y victimización que ocurren reiteradamente entre escolares (Roman \& Murillo, 2011).

Diferentes revisiones sistemáticas referidas a la violencia escolar señalan que ésta debilita la calidad de vida de la población, aumenta el miedo y la inseguridad ciudadana, y deteriora el capital social porque genera aislamiento y desconfianza (ÁlvarezGarcía et al., 2010; Srabstein, Berkman, \& Pyntikova, 2007). Recientemente, un estudio promovido por UNESCO en 15 países de América Latina sobre logros de aprendizaje y factores asociados, concluyó que existe una relación estadísticamente significativa entre el sexo de los estudiantes y el hecho de haber sido víctima de algún tipo de robo, insulto, amenaza o maltrato físico. Asimismo, un estudio sobre bienestar infantil basado en el componente cualitativo de "Niños del Milenio", comparó distintas percepciones y opiniones de escolares contactados en el año 2007 (a la edad de 12, en promedio) y en el año 2011, e identificó que los principales factores que ponen en riesgo su bienestar subjetivo son la pérdida de apoyo de sus padres, ser víctimas de violencia y el sentimiento de soledad (Escobal \& Benites, 2012).

Es posible constatar que uno de los factores de mayor riesgo para el desarrollo y bienestar de los niños y adolescentes es la violencia en su entorno (familiar, escolar y comunitario), la cual se encuentra asociada con menores logros de aprendizaje, problemas de conducta, conductas de riesgo, débil autocontrol, mayor probabilidad de repetir grados, ser expulsados o suspendidos, menor probabilidad de graduarse; trayendo consigo efectos negativos en cuanto al desarrollo psicosocial y el bienestar emocional (López, Morales \& Ayala, 2009; Veltman \& Browne 2001; Zolotor et al. 1999).

Por su parte, cuando la violencia y el acoso se dan al interior de la escuela, afecta negativamente la convivencia escolar y atenta contra la calidad educativa, donde los efectos negativos alcanzan no sólo a las víctimas sino también a los agresores. La evidencia señala que, en contextos de violencia, la escuela juega un rol fundamental en generar factores protectores y promover la resiliencia en niños y adolescentes en condiciones de riesgo, pero esto no se logra efectivamente cuando la violencia se reproduce en la misma escuela. Un estudio realizado en Chile muestra que estudiantes diagnosticados con necesidades educativas especiales reportan 2 veces más agresiones que estudiantes sin este diagnóstico (Villalobos-Parada et al., 2015).

Existen vínculos entre el bullying y distintos indicadores de salud mental como la depresión, la autoestima, el aislamiento y la autorregulación, y supone un nexo con el desempeño académico de los estudiantes. Por ejemplo, la depresión y la ansiedad pueden ocasionar que los niños se concentren menos (Diagne, 2009) mientras que el aislamiento puede hacer que los estudiantes se beneficien en menor medida de los peer-effects dentro de la escuela (Lavy \& Schlosser 2011; Neidell \& Waldfogel, 2010).

Además, la violencia escolar está asociada con el ausentismo y la deserción escolar. Por ejemplo, Kochenderfer \& Ladd (1996) realizan un estudio utilizando un panel de datos de niños de inicial y encuentran evidencia de que aquellos niños que fueron víctimas de bullying presentan mayor tendencia de no querer asistir a clases.

\section{Soporte Social}

La adolescencia es un período crucial de formación, madurez y desarrollo de las personas. Diversos estudios demuestran que el desarrollo de los estudiantes debe promoverse desde una perspectiva comprehensiva, que tomen en cuenta los aspectos físicos y psicológicos dentro de los ambientes familiar, escolar y comunitario (Finkelstein, Von Eye, \& Preece 1994), y evaluaciones ecológicas que sean utilizadas democrática y propositivamente para fortalecer las capacidades de las escuelas (López et al., 2014).

Según el modelo ecológico de Bronfenbrenner \& Ceci (1994), la integración de diversos espacios de interacción de una persona, especialmente en niños y adolescentes, conlleva a reportar mayores niveles de bienestar y menor exposición a ser víctima de violencia. El soporte social modera la reducción de la probabilidad de estar expuesto a violencia, bullying y múltiples tipos de abusos, siendo el soporte familiar y las relaciones positivas en la escuela las que tienen un rol protector frente a la violencia (Strom et al. 2013). Este enfoque integral puede contribuir a promover el bienestar en general y en la escuela, mediante el desarrollo de competencias sociales, emocionales, cognitivas y ciudadanas de quienes conforman la comunidad educativa (Konu \& Lintonen 2006). Existe extensa evidencia empírica que demuestra que el dominio de habilidades sociales del estudiante en la adolescencia está asociado con un mayor bienestar, mejor desempeño escolar (Durlak et al 2011) y el desarrollo positivo de la juventud (Cohen, Pickeral \& Levine, 2010). 


\section{Apoyo familiar}

La dinámica familiar, entendida como el soporte familiar experimentado, hostilidad familiar, tiempo compartido, entre otros, evidencia tener una asociación cuando los estudiantes reportan ser víctimas de bullying. Específicamente, estudiantes que manifiestan haber sido víctima de bullying por sus pares tienen padres que describen sus hogares como espacios donde los miembros de la familia se critican entre sí, tienen pocas reglas y comparten poco tiempo juntos (Holt, Kaufman \& Finkelhor, 2008), espacios en el cuál no se sienten integrados y existe poca identificación o cohesión familiar.

Asimismo, desde modelos derivados de la teoría del aprendizaje social (Bandura, 1973), los factores del contexto familiar están relacionados con la presencia del bullying, en la medida que se considera al clima familiar integra aspectos referentes a las relaciones y al funcionamiento social en general (Moos, Moos \& Trickett, 1984), así como el estrecho vínculo existente entre la calidad de las relaciones familiares y la implicación en conductas violentas (Estevez et al., 2012; Gerard \& Buehler, 1999; Musitu et al., 2006).

\section{Apoyo de Pares}

La percepción de los adolescentes acerca de la calidad del apoyo recibido de sus pares sirve como moderador del impacto de experiencias negativas de diversas maneras. Teóricamente, el contar con amigos cercanos puede proteger a adolescentes de eventos estresantes y/o intimidantes debido al efecto de la calidad de la amistad en aumentar la autoestima, proporciona asistencia para hacer frente y aumenta la seguridad percibida en las relaciones sociales (Sandler, Miller \& Wolchik, 1989).

El apoyo de pares resulta ser una variable clave en programas antibullying (Salmivalli, Kärnä \& Poskiparta, 2010; Ttofi \& Farrington, 2011 ; Salmivalli, 2010; Holt \& Espelage, 2007; Polanin, Espelage \& Pigott, 2012), siendo una de las estrategias de afrontamiento más utilizadas en reducir el bullying, al conectarse con sus pares (Bourke \& Burgman, 2010), debido al efecto amortiguador que tiene pese a las consecuencias negativas que conlleva la violencia (Villalobos-Parada et al., 2016).

Asimismo, la calidad de la amistad percibida resulta ser un moderador en la victimización de pares (Bollmer et al. 2005). Sin embargo, este efecto ha sido débilmente estudiado en estudiantes de transición entre primaria y secundaria, etapa donde se producen situaciones de estrés que puede promover comportamiento agresivo entre pares en un intento de definir su nuevo rol en esta estructura social (Espelage \& Holt, 2001).

\section{Apoyo de escuela}

La escuela resulta una variable importante para comprender el bullying, debido a la interacción que se suscita dentro de ella (Salmivalli et al., 1996). Un clima escolar positivo reduce las tasas de victimización y crea un entorno seguro escolar (Astor, Guerra, \& Van Acker, 2010; Benbenishty \& Astor, 2005; Cornell, Shukla, \& Konold, 2015; Espelage, Gutgsell, \& Swearer, 2004; Benbenishty et al., 2016).

Aun cuando la mayor parte de las investigaciones referidas al apoyo de la escuela sobre los estudiantes se enfoca en el resultado académico, el apoyo social de los profesores, en especial, resulta ser importante y significativo para una mejor salud mental, mayor bienestar, autoestima y valores sociales (Colarossi \& Eccles, 2003; Demaray \& Malecki 2003), incluso puede llegar a ser tanto o más significativo que el apoyo de los pares (Villalobos-Parada et al., 2016). Si los estudiantes perciben que sus profesores son justos y les importan, ellos tienden a desarrollar factores protectores contra la violencia, consumo de alcohol, cigarro y drogas a través del desarrollo de habilidades socioemocionales en el entorno personal, así como una identidad colectiva positiva (McNeely \& Falci, 2004; Santinello, Viendo \& De Vogli, 2011).

\section{Bienestar subjetivo y bullying}

El bienestar subjetivo personal es un constructo multidimensional que involucra un juicio valorativo de su vida en conjunto, así como de diferentes "espacios" o ámbitos de ésta. Considera tanto juicios cognitivos (satisfacción vital con distintos aspectos de la vida que considere importantes) (Tov \& Diener, 2013), como afectivos, entendidos como la balanza de afectos (Jovanović \& Gavrilov-Jerković, 2015), emociones positivas y negativas experimentadas ante determinadas situaciones vitales vividas, las cuales deben mantener un equilibro saludable para no tener efectos nocivos para la salud mental positiva (Diener et al., 2009).

Existe evidencia sobre la influencia de diferentes contextos sociales y culturales sobre el bienestar subjetivo, la cual resulta particularmente importante en la niñez y adolescencia (Diener, Oshi \& Lucas 2003). Asimismo, el bienestar subjetivo está negativamente relacionado con problemas de violencia entre adolescentes. Estudiantes que se sienten inseguros en su escuela, son víctimas de bullying, tienen a reportar menores niveles de bienestar subjetivo (Konu, Lintonen, \& Autio 2002; Rigby 2000), asociado a mayor inseguridad personal, ansiedad, depresión, soledad e infelicidad (Nansel et al. 2001).

Además, estudios sobre bienestar y éxito escolar señalan que ambos se encuentran muy relacionados $y$, a pesar que no se establece que una cause a la otra, 
sí se indica que altos niveles de bienestar subjetivo se relacionan con una mayor probabilidad de éxito escolar, y viceversa (Park, Peterson, \& Sun, 2013).

Nansel et al. (2004) utilizan muestras representativas de estudiantes entre 11 y 16 años, y reporta que las víctimas de violencia escolar poseen dificultades para relacionarse con sus compañeros. Por su parte, Grills \& Ollendick (2002) realizan un estudio con los estudiantes de sexto grado de una escuela en Virginia, Estados Unidos y encuentran que haber sido víctima no solo está muy correlacionado con presentar mayores niveles de depresión y ansiedad interpersonal, sino también con tener baja autoestima y sentirse más inseguro respecto del apoyo que reciben de su entorno social. Hawker \& Boulton (2000) realizaron un meta análisis utilizando estudios de corte transversal elaborados entre los años 1978 y 1997 indican que los episodios de victimización escolar están asociados con menores niveles de bienestar subjetivo en comparación a otros efectos señalados por la literatura como la baja auto estima, el aislamiento o la ansiedad interpersonal.

Este trabajo tiene como propósito presentar nuevos aportes referentes a la dinámica del apoyo social en propias percepciones y evaluaciones de su bienestar. Su propósito consiste en mejorar el bienestar de los niños mediante la creación de conciencia entre los niños, sus padres y sus comunidades, sino también entre líderes de opinión, decisores de políticas, profesionales y público en general.

El proyecto de ISCWeB comenzó el 2010 y, en el mismo año, se aplicó un cuestionario piloto único en Alemania, Brasil, Inglaterra, Israel, Honduras, España y Palestina. En octubre de 2011, de los resultados obtenidos, fue planteada una segunda aplicación. Esta vez considerando tres cuestionarios diferenciados por la etapa de desarrollo de 8,10 y 12 años de edad los niños. Durante 2012 y 2013, este cuestionario se aplicó en 14 países, entre los cuales Alemania, Brasil, Corea del sur, España, Estados Unidos, Honduras, Israel, Reino Unido, Sudáfrica, Turquía y Chile destacan. Más tarde en 2014, la aplicación se extendió a países como Argelia, Colombia, Estonia, Etiopía y Portugal. La aplicación tuvo lugar en las escuelas de cada país, teniendo siempre en cuenta la edad como criterio de inclusión para la muestra.

Tabla 1

Número de estudiantes participante en Brasil y Chile.

\begin{tabular}{lrrll}
\hline & \multicolumn{2}{c}{ Muestra } & \multicolumn{2}{c}{ Edad } \\
\cline { 2 - 5 } & \multicolumn{1}{c}{$\mathrm{n}$} & $\%$ & $\mathrm{M}$ & $\mathrm{SD}$ \\
\hline Brasil & 1,005 & 54,9 & 11,92 & 0,83 \\
Chile & 827,000 & 45,1 & 12,51 & 0,69 \\
Totales & 1,832 & 100,0 & 12,18 & 0,82 \\
\hline
\end{tabular}

diferentes contextos a escolares y sus efectos sobre el bullying y el bienestar subjetivo experimentado en estudiantes de los países de América Latina que fueron parte del estudio internacional sobre bienestar infantil (ISCWeb), en su versión de 12 años.

Diferentes estudios referentes al bullying en niños y adolescentes involucran una serie de factores individuales y de contexto. El enfoque ecológico sirve para comprender de manera holística la interacción de los diferentes espacios del individuo de forma multinivel y su relación con el bullying (Limber 2006) y el bienestar subjetivo (Trujillo, Tovar, Lozano 2004; Limonero et al. 2012; Ramos-Díaz et al. 2015).

\section{MATERIAL Y MÉTODOS}

ElChildrens'Worlds, the International Survey ofChildren's Well-Being (ISCWeB), es un estudio de investigación sobre el bienestar subjetivo de los niños y niñas a nivel mundial. Tiene como objetivo recopilar datos sólidos y representativos sobre la vida y actividades cotidianas, su uso del tiempo de los niños y, en particular en sus
Este estudio consideró estudiantes de Brasil y Chile, y un total de 1832 estudiantes que fueron parte de la aplicación del cuestionario para 12 años. El promedio de edad de los estudiantes es de 12,18 años (DE $=0,82$ ). Se incluyeron diferentes instrumentos en el cuestionario de ISCWeb para niños y niñas de 12 años que fueron usados para este estudio, los cuales son idénticos para ambos países y fueron traducidos y aplicados en sus idiomas correspondientes.

Para el presente estudio se ha considerado para cada una de las variables del modelo el análisis de fiabilidad de la dimensión, a través del cálculo del alfa de Cronbach, para posteriormente realizar un análisis de ecuación estructural, con el propósito de medir la adecuación de estos indicadores para la muestra seleccionada.

A continuación, se detallan los indicadores seleccionados para el modelo en base a los objetivos del estudio:

Apoyo familiar: Calculado a partir de 3 preguntas, cuyo rango de respuesta va de 0 ("totalmente 
Tabla 2

Correlaciones entre las variables del modelo.

\begin{tabular}{lccccc}
\hline Variable 12 años & 1 & 2 & 3 & 4 & 5 \\
\hline 1. Bullying & -- & & & & \\
2. PWI & $-0,181^{* *}$ & -- & & & \\
3. Apoyo familiar & $-0,134^{* *}$ & $0,478^{* *}$ & -- & & \\
4. Apoyo de escuela & $-0,234^{* *}$ & $0,468^{* *}$ & $0,337^{* *}$ & -- & \\
5. Apoyo de amigos & $-0,219^{* *}$ & $0,402^{* *}$ & $0,246^{* *}$ & $0,391^{* *}$ & -- \\
\hline
\end{tabular}

${ }^{*} \mathrm{p}<0,05 \mathrm{y}^{* *} \mathrm{p}<.0,1$ (bilateral)

desacuerdo") a 10 ("totalmente de acuerdo"): (1) Estoy satisfecho con las personas que vivo; (2) Estoy satisfecho con mi vida familiar; (3) Estoy satisfecho con las personas de mi familia. El alfa de Cronbach es de 0,72

Apoyo de escuela: Calculado a partir de 3 preguntas, cuyo rango de respuesta también va de 0 ("totalmente desacuerdo") a 10 ("totalmente de acuerdo"), evalúa la satisfacción del estudiante con: (1) su experiencia en la escuela; (2) su relación con los compañeros de clase; (3) ir a la escuela que asiste. El alfa de Cronbach es de 0,68

Apoyo de amigos: Calculado a partir un único ítem, cuya respuesta va de 0 ("totalmente en desacuerdo") a 10 ("totalmente de acuerdo"), sobre la pregunta ¿Qué tan satisfecho/a estoy de mis amigos?

Bullying: Media de frecuencias de dos preguntas referidas exposición de situaciones de violencia donde 0 ("Nunca") y 3 ("Más de tres veces"). (1) ¿QQue tan seguido te golpea otro niño en la escuela? y (2) ¿Qué tan seguido eres excluido por tus amigos en la escuela? Índice de bienestar personal en la escuela (PWI): escala de Cummins, Eckersley, van Pallant, Vugt \& Misajon (2003), incluye siete ítems relacionados a la satisfacción con la vida en diferentes aspectos de la vida: calidad de vida, salud, logros en la vida, relaciones con otras personas, seguridad personal, cosas materiales y seguridad futura. Estos ítems están expresados en una escala de Likert que va de un rango de 0 ("completamente insatisfecho") a 10 ("completamente satisfecho"). El coeficiente de alfa de Cronbach para este índice es de 0,76.

\section{RESULTADOS}

Las correlaciones son significativas para todas las asociaciones y esperables teóricamente. Para el caso de los apoyos, todos ellos presentan una correlación positiva con el bienestar subjetivo, y para el caso de violencia estas relaciones son negativas. Asimismo, la relación entre bullying y bienestar subjetivo (PWI) también es negativa y significativa.

Al analizar los coeficientes de correlación entre ambos países, estos presentan el mismo patrón comparado con el modelo general, teniendo la misma dirección y nivel de significancia, aunque la fuerza de la correlación difiere.

Para Brasil, existe una correlación negativa entre el bienestar subjetivo y bullying $(r=-0,248)$, la relación de los apoyos con el PWI es positiva y significativa, siendo más importante la relación con la escuela ( $r$ $=0,566)$; y con el bullying todas resultan negativas, destacando la relación, de igual manera, con la escuela $(r=-0,273)$.

Tabla 3

Correlaciones entre las variables del modelo por país.

\begin{tabular}{|c|c|c|c|c|c|}
\hline Variable & 1 & 2 & 3 & 4 & 5 \\
\hline 1. PWI & -- & & & & \\
\hline 2. Bullying & $-0,248^{* *}$ & -- & & & \\
\hline 3. Apoyo familiar & $0,556^{* *}$ & $-0,172^{* *}$ & -- & & \\
\hline 4. Apoyo de escuela & $0,566^{* *}$ & $-0,273^{* *}$ & $0,406^{* *}$ & -- & \\
\hline 5. Apoyo de amigos & $0,480^{* *}$ & $-0,259^{* *}$ & $0,287^{* *}$ & $0,461^{* * *}$ & -- \\
\hline Variable & & & & & \\
\hline 1. PWI & -- & & & & \\
\hline 2. Bullying & $-0,121^{* *}$ & -- & & & \\
\hline 3. Apoyo familiar & $0,417^{* *}$ & $-0,098^{* *}$ & -- & & \\
\hline 4. Apoyo de escuela & $0,390^{* *}$ & $-0,202^{* *}$ & $0,210^{* *}$ & -- & \\
\hline 5. Apoyo de amigos & $0,337^{* *}$ & $-0,173^{* *}$ & $0,285^{* *}$ & $0,338^{* *}$ & - \\
\hline
\end{tabular}

${ }^{*} \mathrm{p}<0,05 \mathrm{y}{ }^{* *} \mathrm{p}<0,01$ (bilateral) 
En el caso de Chile, el PWI y bullying también tiene una relación negativa y significativa $(r=-0,121)$. Comparando con las relaciones sociales, el apoyo familiar representa la variable más importante $(r=$ $0,417)$ sobre la variable PWI. Al igual que en el caso de Brasil, el apoyo de la escuela resulta la más importante con relación al bullying $(r=-0,202)$.

El modelo de ecuaciones estructurales propuesto entre los diferentes tipos de apoyo, bullying y bienestar subjetivo personal cuenta con índices de ajustes adecuados $(\chi 2=558,32, \chi 2 / \mathrm{g} . \mathrm{l} .=6,81, \mathrm{CFI}=0,93$, $\mathrm{TLI}=0,91$ SRMR $=0,061$, RMSEA $=0,056)$. los efectos indirectos no resultan ser significativos para este modelo, tal como se observa en la figura 1.

\section{DISCUSIÓN}

Los resultados permiten confirmar que el apoyo social tiene un efecto positivo en el bienestar subjetivo de los niños y las niñas, tanto a nivel familiar, como escolar y de pares. Las diferencias del efecto que tienen estos tipos de apoyo son de matices, considerando que los participantes de la muestra perciben como más

Tabla 4

Efectos totales reportados por país.

\begin{tabular}{|c|c|c|c|c|}
\hline \multicolumn{3}{|c|}{ Muestra 12 años } & \multirow{2}{*}{$\begin{array}{c}\text { Brasil } \\
--\end{array}$} & \multirow[t]{2}{*}{ Chile } \\
\hline Bullying & $<---$ & Apoyo familiar & & \\
\hline Bullying & $<---$ & Apoyo de escuela & $-0,181^{* *}$ & -- \\
\hline Bullying & $<---$ & Apoyo de amigos & $-0,134^{* *}$ & $0,478^{* *}$ \\
\hline PWI & $<---$ & Apoyo familiar & $-0,234^{* *}$ & $0,468^{* *}$ \\
\hline PWI & $<---$ & Apoyo de escuela & $-0,219^{* *}$ & $0,402^{* *}$ \\
\hline PWI & $<---$ & Apoyo de amigos & & \\
\hline
\end{tabular}

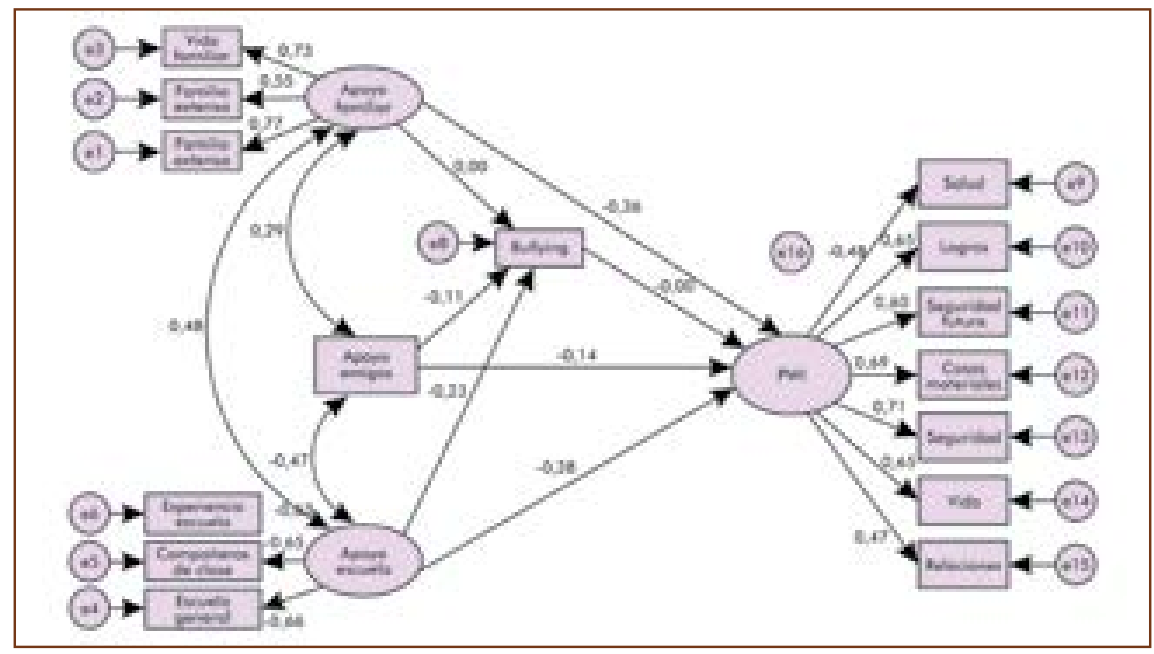

Figura 1, Modelo de Ecuaciones Estructurales.

Nota: Las líneas discontinuas muestran impactos estadísticamente no significativos.

Los resultados del modelo presentado en la tabla 4 muestran que, a nivel general, el apoyo de la escuela $(B=-0,23, p<0,001)$ representa la variable con mayor influencia para disminuir la frecuencia de prevalencia de bullying reportada; seguido por el apoyo de amigos $(B=-0,11$, $p<0,001)$. Referente al efecto de los apoyos sobre el bienestar subjetivo personal, se observa que el apoyo de la escuela $(B=0,38, p<0,001)$, seguido del apoyo familiar $(B=0,36, p<0,001)$ resultan ser los más importantes. Considerando al bullying como variable mediadora entre los apoyos sociales y bienestar subjetivo personal, importante el apoyo de la escuela, seguida del apoyo de la familia y la de pares.

No obstante, es importante considerar que la importancia de estos apoyos es relativa y se relaciona directamente con la edad y la etapa vital en la que se encuentran viviendo, por lo que no es menor tener en cuenta que se trata estudiantes de 12 años. De esta forma, es relevante la diferencia cultural que existe entre países, en la medida que los apoyos no sólo tienen una influencia diferente en función de variables demográficas, sino también culturales. 
Es así como en Brasil se observa que el apoyo más importante, en relación con el bienestar subjetivo, es el escolar, y en Chile, es el familiar.

Por otra parte, al analizar el efecto que tiene el apoyo sobre el bienestar subjetivo, mediado por ser víctima de bullying, se aprecia que a medida que existe mayor apoyo, sí hay menor frecuencia de este hecho al interior de las escuelas. Esto se condice con otras investigaciones presentadas previamente, que constatan el efecto amortiguador de los apoyos sobre los hechos de violencia escolar (Villalobos-Parada et al., 2016).

Cabe destacar que el apoyo social, en distintos niveles, es importante de potenciar en los distintos ámbitos de la vida, en tanto no sólo aumenta el bienestar subjetivo personal, sino también disminuye los hechos de violencia ocurridos, en la medida que se generan espacios de reflexión y comprensión, donde se fomente la confianza para no permitir hechos de violencia y se pueda hacer algo al respecto.

De esta forma, es importante profundizar la manera en cómo son percibidos los distintos apoyos en distintos contextos culturales y en diferentes edades, con el fin de proponer distintas líneas de intervención en apoyo dependiendo de los intereses de los niños, niñas y jóvenes, en función del momento vital en el que se encuentran.

Además, de qué forma distintos programas de intervención basados en apoyos fortalecen el bienestar subjetivo, no sólo a nivel individual, sino también a nivel social, y disminuyen los hechos de violencia, en la medida que se toma conciencia de la forma en cómo esto afecta.

\section{REFERENCIAS BIBLIOGRÁFICAS}

Álvarez-García, D., Rodríguez, C., González-Castro, P., Núñez, J. C., \& Álvarez, L. (2010). La formación de los futuros docentes frente a la violencia escolar. Revista de Psicodidáctica, 15(1), 35-56.

Astor, R. A., Guerra, N., \& Van Acker, R. (2010). How can we improve school safety research? Educational researcher, 39(1), 69-78.

Bandura, A. (1973). Aggression: A social learning analysis. Prentice-Hall.

Benbenishty, R., \& Astor, R. A. (2005). School violence in context: Culture, neighborhood, family, school, and gender. Oxford University Press.

Benbenishty, R., Astor, R. A., Roziner, I., \& Wrabel, S. L. (2016). Testing the Causal Links Between School Climate, School Violence, and School Academic Performance: A Cross-Lagged Panel Autoregressive
Model. Educational Researcher, 45(3), 197-206.

Bollmer, J. M., Milich, R., Harris, M. J., \& Maras, M. A. (2005). A friend in need the role of friendship quality as a protective factor in peer victimization and bullying. Journal of interpersonal violence, 20(6), 701-712.

Bourke, S., \& Burgman, I. (2010). Coping with bullying in Australian schools: how children with disabilities experience support from friends, parents and teachers. Disability \& Society, 25(3), 359-371.

Bronfenbrenner, U., \& Ceci, S. J. (1994). Nature-nuture reconceptualized in developmental perspective: A bioecological model. Psychological review, 101(4), 568.

Cohen, J., Pickeral, T., \& Levine, P. (2010). The foundation for democracy: Promoting social, emotional, ethical, cognitive skills and dispositions in K-12 schools. Inter-American Journal of Education for Democracy, 3(1), 73-94.

Colarossi, L. G., \& Eccles, J. S. (2003). Differential effects of support providers on adolescents' mental health. Social Work Research, 27(1), 19-30.

Cornell, D., Shukla, K., \& Konold, T. (2015). Peer victimization and authoritative school climate: A multilevel approach. Journal of Educational Psychology, 107(4), 1186.

Cummins, R. A., Eckersley, R., Pallant, J., Van Vugt, J., \& Misajon, R. (2003). Developing a national index of subjective wellbeing: The Australian Unity Wellbeing Index. Social indicators research, 64(2), $159-190$

Demaray, M. K., \& Malecki, C. K. (2003). Perceptions of the frequency and importance of social support by students classified as victims, bullies, and bully/victims in an urban middle school. School Psychology Review, 32(3), 471-490.

Diagne, D. (2009). School violence: Evidence from the economics literature and related disciplines. Schweizerische Zeitschrift für Bildungswissenschaften, 31(1), 135-150.

Diener, E. (2000). Subjective well-being: The science of happiness and a proposal for a national index. American psychologist, 55(1), 34.

Diener, E., Oishi, S., \& Lucas, R. E. (2003). Personality, culture, and subjective well-being: Emotional and cognitive evaluations of life. Annual review of psychology, 54(1), 403-425.

Diener, E., Wirtz, D., Tov, W., Kim-Prieto, C., Choi, D., Oishi, S., \& Biswas-Diener, R. (2009). New Wellbeing Measures: Short Scales to Assess Flourishing and Positive and Negative Feelings. Social Indicators Research, 97(2), 143-156. http://doi. org/10.1007/s $11205-009-9493-y$

Durlak, J. A., Weissberg, R. P., Dymnicki, A. B., Taylor, R. D., \& Schellinger, K. B. (2011). The impact of enhancing students' social and emotional learning: A meta-analysis of school-based universal interventions. Child development,82(1), 405-432.

Escobal, J., \& Benites, S. (2012). Algunos impactos del 
programa JUNTOS en el bienestar de los niños: Evidencia basada en el estudio Niños del Milenio.

Espelage, D. L., Gutgsell, E. W., \& Swearer, S. M. (Eds.). (2004). Bullying in American schools: A social-ecological perspective on prevention and intervention. $1^{\circ}$ Edition. Routledge.

Espelage, D. L., \& Holt, M. K. (2001). Bullying and victimization during early adolescence: Peer influences and psychosocial correlates. Journal of Emotional Abuse, 2(2-3), 123-142.

Estévez, E., Inglés, C. J., Emler, N. P., MartínezMonteagudo, M. C., \& Torregrosa, M. S. (2012). Análisis de la relación entre la victimización y la violencia escolar: El rol de la reputación antisocial. Psychosocial Intervention, 21(1), 53-65.

Finkelstein, J. W., Von Eye, A., \& Preece, M. A. (1994). The relationship between aggressive behavior and puberty in normal adolescents: A longitudinal study. Journal of Adolescent Health, 15(4), 319-326.

Gerard, J. M., \& Buehler, C. (1999). Multiple risk factors in the family environment and youth problem behaviors. Journal of Marriage and the Family, 343-361.

Grills, A. E., \& Ollendick, T. H. (2002). Peer victimization, global self-worth, and anxiety in middle school children. Journal of clinical child and adolescent psychology, 31(1), 59-68.

Jovanović, V., \& Gavrilov-Jerković, V. (2015). The Structure of Adolescent Affective Well-Being: The Case of the PANAS Among Serbian Adolescents. Journal of Happiness Studies. http://doi. org/10.1007/s10902-015-9687-8

Hawker, D. S., \& Boulton, M. J. (2000). Twenty years' research on peer victimization and psychosocial maladjustment: a meta-analytic review of crosssectional studies. Journal of child psychology and psychiatry, $41(4), 441-455$.

Holt, M. K., Kaufman Kantor, G., \& Finkelhor, D. (2008). Parent/child concordance about bullying involvement and family characteristics related to bullying and peer victimization. Journal of School Violence, 8(1), 42-63.

Holt, M. K., \& Espelage, D. L. (2007). Perceived social support among bullies, victims, and bully-victims. Journal of Youth and Adolescence, 36(8), 984-994.

Kochenderfer, B. J., \& Ladd, B. (1996). Peer victimization: Cause or consequence of children's school adjustment difficulties. Child Development,67, 1293-1305.

Konu, A., \& Lintonen, T. (2006). Theory-based survey analysis of well-being in secondary schools in Finland. Health Promotion International, $21(1), 27-$ 36.

Konu, A. I., Lintonen, T. P., \& Autio, V. J. (2002). Evaluation of well-being in schools-a multilevel analysis of general subjective well-being. School Effectiveness and School Improvement, 13(2), 187 200.

Lavy, V., \& Schlosser, A. (2011). Mechanisms and impacts of gender peer effects at school. American Economic Journal: Applied Economics, 3(2), 1-33.

Le, A. T., Miller, P. W., Heath, A. C., \& Martin, N. (2005). Early childhood behaviours, schooling and labour market outcomes: Estimates from a sample of twins. Economics of Education Review, 24(1), 1-17.

Limber, S. P. (2006). The Olweus bullying prevention program: An overview of its implementation and research basis. Handbook of school violence and school safety: From research to practice, 293-307.

Limonero, J. T., Tomás-Sábado, J., Fernández-Castro, J., Gómez-Romero, M. J., \& Ardilla-Herrero, A. (2012). Estrategias de afrontamiento resilientes y regulación emocional: predictores de satisfacción con la vida. Psicología conductual, 20(1), 183.

López, V., Ascorra, P., Bilbao, M. de los A., Carrasco, C., Morales, M., Villalobos, B. y Ayala del Castillo, A. (2014). Monitorear la convivencia escolar para fortalecer (no disminuir) las capacidades de las escuelas. Revista Iberoamericana de Evaluación Educativa,6 (2), 201-219

McNeely, C., \& Falci, C. (2004). School connectedness and the transition into and out of Health-Risk behavior among adolescents: A comparison of social belonging and teacher support. Journal of School Health, 74(7), 284-292.

Moos, R. M., Moos, B. S., \& Trickett, E. J. (1984). FES, WES y CES Escalas de Clima Social. Madrid: TEA Ediciones.

Musitu Ochoa, G., Martínez Ferrer, B., \& Murgui Perez, S. (2006). Conflicto marital, apoyo parental y ajuste escolar en adolescentes. Anuario de Psicología, 37(3), 247-258.

Nansel, T. R., Craig, W., Overpeck, M. D., Saluja, G., \& Ruan, W. J. (2004). Cross-national consistency in the relationship between bullying behaviors and psychosocial adjustment. Archives of pediatrics \& adolescent medicine, 158(8), 730-736.

Nansel, T. R., Overpeck, M., Pilla, R. S., Ruan, W. J., Simons-Morton, B., \& Scheidt, P. (2001). Bullying behaviors among US youth: Prevalence and association with psychosocial adjustment. JAMA, 285(16), 2094-2100.

Naylor, P., \& Cowie, H. (1999). The effectiveness of peer support systems in challenging school bullying: the perspectives and experiences of teachers and pupils. Journal of adolescence, 22(4), 467-479.

Neidell, M., \& Waldfogel, J. (2010). Cognitive and noncognitive peer effects in early education. The Review of Economics and Statistics, 92(3), 562576.

Organización Mundial de la Salud. (2003). Informe mundial sobre la violencia y la salud.

Polanin, J. R., Espelage, D. L., \& Pigott, T. D. (2012). A meta-analysis of school-based bullying prevention programs' effects on bystander intervention behavior. School Psychology Review, $41(1), 47$.

Ramos-Díaz, E., Rodríguez-Fernández, A., FernándezZabala, A., Revuelta, L., \& Axpe, I. (2016). 
Resiliencia y bienestar subjetivo en función del sexo y del nivel educativo en la adolescencia. European Journal of Child Development, Education and Psychopathology, 3(1).

Rigby, K. E. N. (2000). Effects of peer victimization in schools and perceived social support on adolescent well-being. Journal of Adolescence, 23(1), 57-68.

Rogers, B. (2015). Classroom behaviour: A practical guide to effective teaching, behaviour management and colleague support. Sage.

Román, M. E., \& Torrecilla, F. J. M. (2011). América Latina: violencia entre estudiantes y desempeño escolar. Revista de la CEPAL, (104), 37-54.

Salmivalli, C. (2010). Bullying and the peer group: A review. Aggression and Violent Behavior, 15(2), 112-120.

Salmivalli, C., Kärnä, A., \& Poskiparta, E. (2010). From peer putdowns to peer support: A theoretical model and how it translated into a national anti-bullying program. In S. R. Jimerson, S. M. Swearer \& D. L. Espelage (Hrsg). Handbook of Bullying in Schools: An International Perspective. New York: Routledge.

Salmivalli, C., Lagerspetz, K., Björkqvist, K., Österman, K., \& Kaukiainen, A. (1996). Bullying as a group process: Participant roles and their relations to social status within the group. Aggressive behavior, 22(1), 1-15.

Sandler, I. N., Miller, P., Short, J., \& Wolchik, S. A. (1989). Social support as a protective factor for children in stress. Children's social networks and social supports, 277-307.

Santinello, M., Vieno, A., \& De Vogli, R. (2011). Bullying in Italian schools: the role of perceived teacher unfairness. European journal of psychology of education, 26(2), 235-246.

Srabstein, J. C., Berkman, B. E., \& Pyntikova, E. (2008). Antibullying legislation: A public health perspective. Journal of Adolescent Health, 42(1), 11-20.

Strøm, I. F., Thoresen, S., Wentzel-Larsen, T., \& Dyb, G. (2013). Violence, bullying and academic achievement: A study of 15-year-old adolescents and their school environment. Child Abuse \& Neglect, 37(4), 243-251.

Tov, W., \& Diener, E. (2013). Subjective Well-being. In K. Keith (Ed.), Encyclopedia of Cross-Cultural Psychology (pp. 1-7). New York: Wiley.

Trujillo, S., Tovar, C., \& Lozano, M. (2004). Formulación de un modelo teórico de la calidad de vida desde la psicología. Universitas Psychologica, 3(1), 8998.

Ttofi, M. M., \& Farrington, D. P. (2011). Effectiveness of school-based programs to reduce bullying: A systematic and meta-analytic review. Journal of Experimental Criminology, 7(1), 27-56.

Veltman, M. W., \& Browne, K. D. (2001). Three decades of child maltreatment research Implications for the School Years. Trauma, Violence, \& Abuse, 2(3), 215-239.

Villalobos-Parada, B., Carrasco, C., Olavarría, D., Ortiz, S., López, V., Oyarzún, D., Ascorra, P., Ayala, Bilbao, Morales, M., \& Álvarez, J. (2016). Victimización de Pares y Satisfacción con la Vida: La Influencia del Apoyo de Profesores y Compañeros de la Escuela. Psykhe, 25(2), xx-xx.

Villalobos-Parada, B., López, V., Bilbao, M.A., Carrasco, C., Ascorra, P., Morales, M., Álvarez, J., AyalaDel Castillo, A., Olavarría, D. \& Ortiz, S. (2015). Victimización del personal de la escuela hacia estudiantes referidos a proyectos de integración escolar. Psychology, Society \& Education. Vol. 7(2), 133-152.

Zeedyk, M. S., Gallacher, J., Henderson, M., Hope, G., Husband, B., \& Lindsay, K. (2003). Negotiating the transition from primary to secondary school perceptions of pupils, parents and teachers. School Psychology International,24(1), 67-79.

Zolotor, A., Kotch, J., Dufort, V., Winsor, J., Catellier, D., \& Bou-Saada, I. (1999). School performance in a longitudinal cohort of children at risk of maltreatment. Maternal and Child Health Journal, $3(1), 19-27$. 